\title{
Improvement of the properties of multilayer coextruded films by control of the interphase characteristics
}

\author{
François ROUSSET, Patrick BOURGIN and Liviu-Iulian PALADE \\ Laboratoire de Recherche Pluridisciplinaire en Plasturgie (ERT $\left.n^{\circ} 10\right)$ \\ Site de Plasturgie de l'INSA de Lyon, BP 807 - 01108 Oyonnax Cedex France \\ TEL:0033-4-74-81-93-20 FAX:0033-4-74-81-93-01 e-mail:patrick.bourgin@insa-lyon.fr \\ (Received 13, October 2004 Accepted 31, January 2005)
}

\begin{abstract}
Coextrusion technologies are commonly used to produce multilayered composite sheets of films. Each layer of these composite materials provides a specific end-use characteristic, suc as optical, mechanical or barrier properties. Processing together polymers of different natures may have two consequences : on the one hand, the flow of two adjacent different liquids may give rise to interfacial instabilities which are detrimental to the quality of the final product. On the other hand, the difference of chemical natures of the polymers may lead to delamination of the multilayer film. In order to avoid the latter problem, polymers are compatibilized by introducing a binder between adjacent layers. As a consequence, a non-zero thickness interphase is created between the layers. In this paper, it is theoretically analysed how this interphase may influence the flow stability. As a matter of fact, it is experimentally known that flows of compatible polymers are more stable than those of incompatible systems. Next, a way to optimize the process is proposed.
\end{abstract}

Keywords : Coextrusion, Interfacial Instability, Compatible polymers, Interdiffusion, Energy dissipation

\section{INTRODUCTION}

Coextrusion is an industrial process used to form multilayered sheets of films which are suitable for various applications ranging from food packaging to reflective polarizers. The main problem is to process together polymers of different rheological properties. It is well known that under certain operating conditions, wavy interfaces can be observed inside the die. This interfacial instability affects the quality of the final product. In addition, the use of polymers of different chemical natures may eventually lead to delamination of the layers. The adjacent layers have therefore to be stuck together. For so doing, a binder is classically introduced to form a compatible system, the geometrical interface being replaced by an interphase of finite thickness. However, coextrusion flows of two compatible polymers are generally known to be more stable than the same flows of incompatible systems. As a first step we try to explain the low disturbance growth rate of compatible systems by the sole presence of this intermediate layer. However, this approach fails to explain the modification of the flow stability map. As a second step, we formulate an energetic approach of the problem. A modified growth rate is then determined by taking into account the energy dissipated by the interdiffusion process. This second approach enables us to explain the increase of the stable domain size in the case of compatible polymer systems. 


\section{MECHANICAL APPROACH}

Yih ${ }^{1}$ first studied the stability of Poiseuille flows of two Newtonian immiscible fluids submitted to very long waves. Using linear stability theory he showed that a viscosity difference could lead to instability, even for low Reynolds numbers. His analysis was extended by numerous authors to other situations. All these investigations show that the interfacial stability of multilayer flows is determined by a number of factors which can be either essential (e.g., thickness, viscosity and elasticity ratios) or not very influential (e.g., density ratios and interfacial tensions).

\section{II-A. Problem statement}

In this section we want to determine whether the sole presence of an inner thin layer is able to influence the stability of the flow of a compatible polymeric system. For so doing, we consider a three-layer Poiseuille flow (in which layers 1, 2 and 3 are occupied respectively by polymer $\mathrm{A}$, the interphase and polymer B) through an infinite parallel plate channel geometry, as shown in Fig. 1.

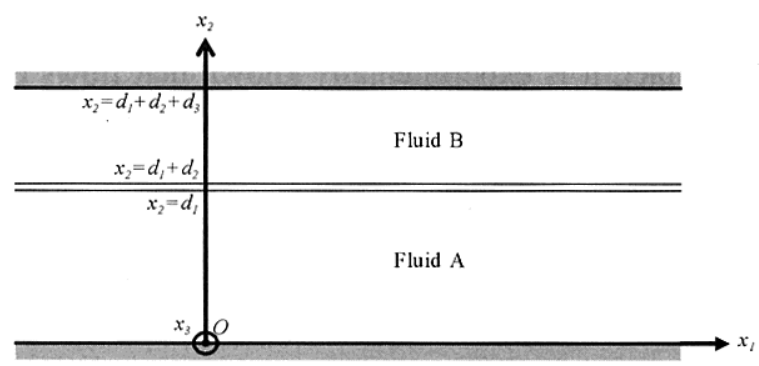

Fig.1. Definition sketch.

Each fluid is supposed to be incompressible and Newtonian. Thus, in each layer $k(k=1,2,3)$, the governing equations are the incompressibility and the Navier-Stokes equations. Let us note that in this model, the viscosity and the thickness of the interphase are neither function of time nor of space variables. In this section we consider indeed that the interphase is already formed. Moreover, the density is assumed to be the same in each layer as the effect of gravity is not studied. This system is completed by the no-slip boundary condition and the continuity conditions at the interfaces on velocity and stress tensor.

\section{II-B. Perturbation flow}

The undisturbed flow (or primary flow) is a channel flow with parabolic velocity profiles. An infinitesimal disturbance is applied to the primary flow to study the stability of the interphase. The motion is merely the sum of the primary motion and the perturbation motion as shown in Eq. 1 .

$$
\begin{gathered}
u_{j, k}=\bar{u}_{j, k}+\widetilde{u}_{j, k} \\
p=\bar{p}+\tilde{p}
\end{gathered}
$$

where bars (resp. tildas) over variables correspond to undisturbed (resp. disturbed) variables. In our stability analysis we assume the validity of Squire's theorem, ${ }^{2}$ namely the two-dimensional disturbances are more unstable than three-dimensional ones, and we therefore consider two-dimensional disturbances. The classical representation of the velocity components (expressed in terms of a stream function) and the disturbed pressures in the linear stability analysis has an exponential form:

$$
\left\{\begin{aligned}
\psi_{k}\left(x_{1}, x_{2}, t\right) & =\phi_{k}\left(x_{2}\right) e^{i \alpha\left(x_{1}-c t\right)} \\
\tilde{p}\left(x_{1}, x_{2}, t\right) & =f\left(x_{2}\right) e^{i \alpha\left(x_{1}-c t\right)}
\end{aligned}\right.
$$

where $\cdot$ is the (real) wave number and $c$ is the complex velocity of the disturbance. By substituting the perturbed variables into motion equations, one obtains the Orr-Sommerfeld equations:

$$
\begin{aligned}
\phi_{k}^{\prime V} & -2 \alpha^{2} \phi_{k}^{\prime \prime}+\alpha^{4} \phi \\
& =i \alpha \frac{R e}{m_{k}^{2}}\left[\left(\phi_{k}^{\prime \prime}-\alpha^{2} \phi_{k}\right)\left(\bar{u}_{1, k}-c\right)-\bar{u}_{1, k}^{\prime \prime} \phi_{k}\right]
\end{aligned}
$$

where $R e$ is the Reynolds number, $m_{k}$ is the viscosity ratio defined by $m_{k}=\mu_{k} / \mu_{1}$, and where the derivatives with respect to $x_{2}$ are denoted by primes. The interfacial boundary conditions need to be evaluated at the disturbed locations of the interfaces. These interfacial conditions about the steady-state interfacial positions are then expressed through Taylor series. The Orr-Sommerfeld equations, the boundary conditions and the interfacial conditions form an eigenvalue problem in which $c$ is the eigenvalue and ${ }_{k}$ the eigenfunctions. The problem is solved by using a non-singular perturbation for in the vicinity of 0 , which corresponds to the case of very long waves. Hence, the eigenfunctions and the eigenvalues are expanded as a regular perturbation 
series of as follows shown in Eq. 4.

$$
\begin{gathered}
\phi_{k}=\phi_{k, 0}+\alpha \phi_{k, 1}+O\left(\alpha^{2}\right) \\
c=c_{0}+\alpha c_{1}+O\left(\alpha^{2}\right)
\end{gathered}
$$

The calculation lead to two zeroth-order eigenvalues, which are found to be real and correspond to the amplification factors of waves. These two phase velocities give two possible modes of wave motion. We then determine the first-order solutions, as the stability of the flow is determined by the sign of the imaginary part of $c$ (the flow is respectively stable, unstable or neutrally stable according to whether $\operatorname{Im}(c)$ is negative, positive or zero). We obtain two first-order eigenvalues which are purely imaginary. Therefore, the stability conditions are determined by the sign of $c_{l, a}$ and $c_{1, b}$. We now have to take into account the fact that $d_{2}$ (i.e. thickness of the inner layer) is negligible as compared to $d_{1}$ and $d_{3}$. Thus the two eigenvalues are expanded and approximated by the following Taylor series:

$$
\begin{gathered}
c_{1, a}=i \operatorname{ReJ}_{l, a}+O\left(d_{2}\right) \\
c_{1, b}=i \operatorname{ReJ}_{l, b}\left(d_{2}^{2}\right)+O\left(d_{2}^{3}\right)
\end{gathered}
$$

where $J_{l, a}$ and $J_{l, b}$ are functions of thickness and viscosity ratios which result from straightforward but cumbersome calculations. Scotto ${ }^{3}$ showed that:

$$
\operatorname{sign}\left(J_{1, b}\right)=\operatorname{sign}\left(J_{1, a}\right) \times\left(\eta_{3}-\eta_{2}\right)\left(\eta_{2}-\eta_{1}\right)
$$

As the inner layer is supposed to represent an interphase, we can assume that its viscosity is intermediate between the one of the two outer layers. Thus, $J_{l, a}$ and $J_{l, b}$ are always of the same sign. As a consequence, the signs of $c_{1, a}$ and $c_{1, b}$ are always the same as the one of the eigenvalue of the two-fluid flow made of the two outer fluids. The stability criteria of the two-layer Poiseuille flow composed by the two outer layers are similar to those proposed by Yiantsios and Higgins ${ }^{4}$ as:

$$
\operatorname{sign}\left(c_{1, a}\right)=\operatorname{sign}\left(\left(m_{3}-1\right)\left(m_{3}-\varepsilon_{3}^{2}\right)\right)
$$

where $\varepsilon_{3}=d_{3} / d_{1}$.

It means that the less viscous fluid has to be in the thinner layer in order the flow to be stable.

As the stability criteria for the three-layer flow are similar to theese for the two-layer flow, we can say that the reduction in the effective viscosity ratio does not affect the stability criteria of the flow. It means that if the two-layer flow is unstable (respectively stable), the three-layer flow will remain unstable (resp. stable). As a conclusion, the reduction in the effective viscosity ratio enables to explain the diminished growth rates observed for flows of compatible fluids but not the reduction in the size of the unstable region. For that purpose, we need a complementary approach in which energetic aspects of the formation of the interphase are taken into account in the stability analysis.

\section{ENERGETIC APPROACH}

Very comprehensive experiments have been carried out by Wilson and Khomami. ${ }^{5-7}$ The authors found a good agreement between theoretical and experimental growth rates in the cas of immiscible fluids. However, considering a two-polymer system with interfacial diffusion (i.e. compatible polymers), they found that experimental growth rates are lower than those predicted for immiscible polymers. The authors proposed two mechanisms to explain this weak sensitivity to disturbances: reduction in the effective viscosity ratio and energy dissipation at the interphase. Having previously shown that there is apparently no strong viscosity effect, we now propose an energetic approach.

\section{III-A. Problem statement}

The section devoted to the energetic approach falls into three parts. As a first step we evaluate $\dot{E}_{k}$ which is the rate of change of the kinetic energy of the disturbance in layer $k$. Finally, we determine the mechanical energy of the global flow $\dot{E}$ and normalize it so that:

$$
\dot{E}=\alpha \operatorname{Im}(c)
$$

In a second step the energy dissipated by the interdiffusion process $\dot{E}_{i}$ is determined. A way to evaluate $\dot{E}_{j}$ is to examine the adhesive bond strength of the final product. According to Kim and $\mathrm{Han}^{8}$ who proposed the following expression to evaluate $G$ under a shear flow field (considering a Fickean one-dimensional diffusion) is as follows:

$$
G \propto\left(t \alpha_{a v g}|\chi|\right)^{1 / 4}
$$

where $x$ is the interaction parameter, $t$ the diffusion time, $\alpha_{\text {avg }}$ the average orientation factor The latter 
factor depends on the steady-state shear compliance of the polymers and on shear stress at the interface. Then, the energy dissipated by the interdiffusion process $\dot{E}_{i}$ is normalized so that at rest (i.e. for a null shear stress at the interface):

$$
\dot{E}_{i}=G=n \varepsilon d
$$

where, according to Wu et al. ${ }^{9}$, " $n$ is the number of chains bridging the original interface per unit area, $\varepsilon$ the attractive energy per unit length of interpenetrating chain, and $d$ the root-mean-square interpenetration depth (i.e. the interfacial thickness)".

The energy available for the growth of the wave $\dot{E}_{c}$ is finally given by:

$$
\dot{E}_{c}=\dot{E}-\dot{E}_{i}
$$

\section{III-B. Illustrative examples}

Let us first consider a plane Poiseuille flow of two incompatible polymers $(\chi=0)$ submitted to very long waves. With the previous notations, the flow is stable if $J_{l, a}\left(\varepsilon_{3}, m_{3}\right)<0$ and the energy available for the growth of the wave is given by:

$$
\dot{E}=\alpha \cdot \operatorname{Im}(c)=\alpha \cdot R e \cdot J_{1, a}\left(\varepsilon_{3}, m_{3}\right)
$$

It means that the stability of the flow is only determined by $\varepsilon_{3}$ and $m_{3}$ whereas the energy available for the growth is also a function of the Reynolds number.

Let us now consider that some interdiffusion process takes place between the two polymers. The energy dissipated by interdiffusion $\dot{E}_{i}$ is not only a function of $\varepsilon_{3}$ and $m_{3}$ but also of the Reynolds number and the interaction parameter. The energy available for the growth of the wave can be expressed by:

$$
\dot{E}_{c}=\dot{E}-E_{i}=\alpha \cdot \operatorname{Re} \cdot J_{l, a}\left(\varepsilon_{3}, m_{3}\right)-\dot{E}_{i}\left(\varepsilon_{3}, m_{3}, R e, \chi\right)
$$

We can then determine a modified growth rate by taking into account the energy dissipated by the interdiffusion process:

$$
\operatorname{Im}\left(c_{c}\right)=\dot{E}_{c} / \alpha
$$

In the following figures (Figs. 2-4) parameter $\operatorname{Im}\left(c_{c}\right) / R e$ is represented as a function of $\varepsilon_{3}$ and $m_{3}$ for several values of $R e$ and $\chi$. The reference will be given by Fig. 2 which corresponds to the case $\chi=0$. Indeed, the stability map presents the same shape for any Reynolds number when there is no interdiffusion.
Let consider for example an extrusion die with the following dimensions: height $\mathrm{H}=10^{-3} \mathrm{~m}$ and length $\mathrm{L}=10^{-2} \mathrm{~m}$. We also assume that each polymer presents a density of $10^{3} \mathrm{~kg} / \mathrm{m}^{3}$ and a steady-state shear compliance of $10^{-5} \mathrm{~Pa}^{-1}$. The viscosity of layer 1 is set to be $10^{4} \mathrm{~Pa}$.s and the wavenumber of the disturbance $\alpha$ is set to be equal to 10 . We first consider the case of a very weak - but realistic Reynolds number $\left(\operatorname{Re}=10^{-5}\right)$.

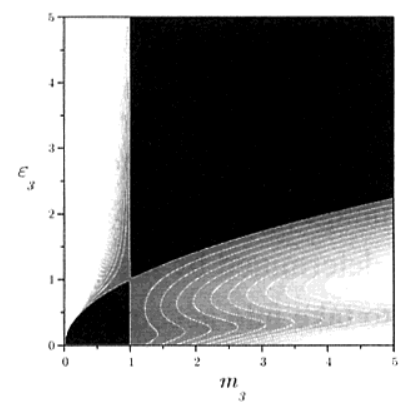

Fig. 2. Stability map in the case $\chi=0$.

Black zones correspond to stable regions and grey zones to unstable regions.

Figs. 3(a) and 3(b) correspond respectively to the cases $\chi=-0.1$ and $\chi=-0.7$, the latter characterizing a very strong chemical affinity. We note that taking into account the interdiffusion process enlarges the stable domains, which is consistent with Wilson and Khomami's observations.
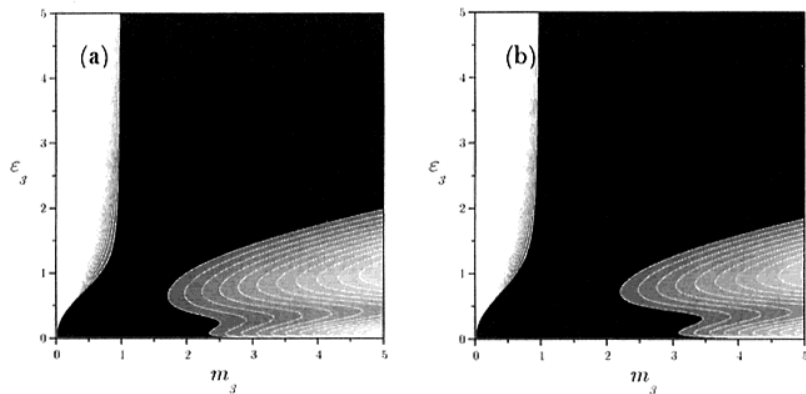

Fig. 3. Stability maps for $\mathrm{Re}=10-5$ and

$$
\chi=-0.1 \text { (a) } \chi=-0.7 \text { (b). }
$$

Let us now consider a larger value for the Reynolds number, say $R e=10^{-3}$. Figs. 4(a) and 4(b) correspond respectively to cases $\chi=-0.1$ and $\chi=-0.7$. For this value of the Reynolds number, taking into account interdiffusion phenomena has no significant effect on the stability maps. Indeed, at 
higher Reynolds numbers, more energy is available for the growth of the wave and less energy is dissipated by interdiffusion.
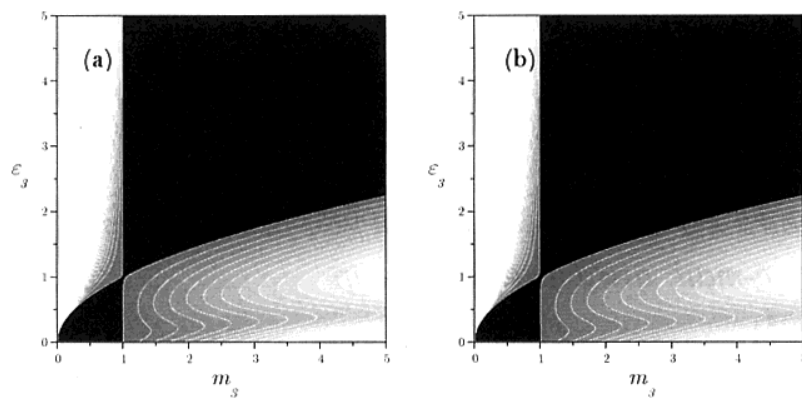

Fig. 4. Stability maps for $\mathrm{Re}=5 \cdot 10-4$ and $\chi=-0.1$ (a) $\chi=-0.7$ (b).

\section{CONCLUSION}

In this study we proposed a way to explain why stratified flows of compatible polymers are generally more stable than the same flows of incompatible polymers. We first showed that taking into account an interphase between the polymers (i.e. considering the reduction in the effective viscosity ratio) enables to explain the diminished growth rates observed but not the reduction of the unstable region. We then investigated the reduction of available energy due to mixing and diffusion processes. This second approach enables us to explain the reduction of the unstable regions at low Reynolds numbers. However it should be noted that the interaction parameter has a weak effect on stability maps. In addition one must keep in mind that the two phenomena (reduction in the effective viscosity ratio and removal of wave energy) take place simultaneously and both tend to damp down the waves initially imposed to the flow. Further investigations will be dedicated to moderate wavelengths and to a viscoelastic model for the fluid. A way to evaluate the energy dissipated by the diffusion process through statistical considerations is in progress.

\section{ACKNOWLEDGEMENTS}

This research was supported by the "Ministère de l'Éducation Nationale, de la Recherche et de la Technologie" (MENRT, France). A doctoral grant was awarded by the MENRT to F.R. We also acknowledge Mr. Maël Moguedet for computer assistance.

\section{REFERENCES}

${ }^{1} \mathrm{YIH}, \quad$ C.-S., Instability due to viscosity stratification, J. Fluid Mech., 27 (2), pp. 337-352, 1967.

${ }^{2}$ SQUIRE, H.B., On the stability of three-dimensional disturbances of viscous flow between parallel walls, Proc. Roy. Soc. A, 142, pp. 621, 1933.

${ }^{3}$ SCOTTO, S., Étude de stabilité des écoulements multicouches de fluides non newtoniens, $\mathrm{PhD}$ Thesis , Université de Nice - Sophia Antipolis, 1998.

${ }^{4}$ YIANTSIOS, S.G., HIGGINS, B.G., Linear stability of plane Poiseuille flow of two superposed fluids, Phys. Fluids, 31 (11), pp. 3225-3238, 1988.

${ }^{5}$ WILSON, G.M., KHOMAMI, B., An experimental investigation of interfacial instabilities in multilayer flow of viscoelastic fluids. Part I. Incompatible polymer systems, J. of Non-Newt. Fluid Mech., 45, pp.355-384, 1992.

${ }^{6}$ WILSON, G.M., KHOMAMI, B., An experimental investigation of interfacial instabilities in multilayer flow of viscoelastic fluids. Part II. Elastic and nonlinear effects in compatible polymer systems, J. Rheol., 37 (2), pp. 315-339, 1993a.

${ }^{7}$ WILSON, G.M., KHOMAMI, B., An experimental investigation of interfacial instabilities in multilayer flow of viscoelastic fluids. Part III. Compatible polymer systems, $J$. Rheol., 37 (2), pp. 341-354, 1993b.

${ }^{8}$ KIM, J.K., HAN, C.D., Polymer-polymer interdiffusion during coextrusion, Polym. Eng. Sci., 31 (4), pp. 258-269, 1991.

${ }^{9}$ WU, S., CHUANG, H.K., HAN, C.D., Diffuse interface between polymers: Structure and kinetics, J. Polymer Sci., 24, pp. 143-159, 1986.

Presented at $5^{\text {th }}$ Japan France Seminar on Intelligent Materials and Structures (October 13-15, 2004 ) 